\title{
Gender Differences in Usage of Social Networking Sites and Perceived Online Social Support on Psychological Well Being of Youth
}

\author{
Nishi Misra ${ }^{1}$, Shivani Dangi ${ }^{1}$, Sanaya Patel $^{2}$
}

\section{ABSTRACT}

Social Networking Sites have been consuming a major part in the Youth daily routine. The study was conducted with an aim to study the effect of social networking sites and perceived social support on the psychological well-being of the youth. The sample size was 100 which had 50 males and 50 females. All the participants were college going students or new entrants in the corporate sector. The participants were asked to fill the questionnaire which had four parts -1 . Social Networking Sites Survey, 2. Multidimensional Scale of Perceived Social Support Assessment, 3. Psychological General Well-Being Index. The results indicated a negative relationship between the usage of social networking sites and the psychological well-being while positive relationship existed between online perceived social support and psychological wellbeing. Certain gender differences were also found among the variables like perceived social support and attraction towards social networking sites. This study has a lot of implications in terms of creating awareness among the youth so that they limit the usage of social networking sites and also a lot of research in the Indian context is yet to be done.

Keywords: Social Networking Sites, Online Perceived social support, Psychological well being

Online social networking and Internet communication is becoming wildly popular with adolesc ents and young adults (Allen, Evans, Hare, Mikami, \& Szwedo, 2010, AnderonButcher, Ball, Br zozowski, Lasseigne, Lehnert, \& McCormick, 2010; DeGroot, Ledbetter, Mao, Mazer, Meyer, \& Swafford, 2011; Finkelhor, Mitchell, \& Wolack, 2002; Greenfield \& Subrahmanyam, 2008; Kra mer \& Winter, 2008; Regan \& Steeves, 2010; Sheldon, 2008). According to the Nielsen Company, global consumers spent more than five and a half hours on social networking sites like Face book and Twitter in December 2009, an 82\% increase from the same time in the previous year when users were spending just over three hours on social networking sites.

\footnotetext{
${ }^{1}$ Defence Institute of Psychological Research, Defence R\&D Organisation, Delhi, India

${ }^{2}$ Deen Dayal Upadhyaya Petroleum University, Gandinagar, Delhi, India

(C) 2015 I N Misra, S Dangi, S Patel; licensee IJIP. This is an Open Access Research distributed under the terms of the Creative Commons Attribution License (http://creativecommons.org/licenses/by/2.0), which permits unrestricted use, distribution, and reproduction in any Medium, provided the original work is properly cited.
} 


\section{Gender Differences in Usage of Social Networking Sites and Perceived Online Social Support on Psychological Well Being of Youth}

\section{SOCIAL NETWORKING SITES (SNSS):}

Social networking phenomenon has emerged since the last 15-20 years. Since that time, SNS has grown from a niche to a mass online activity, in which millions of internet users are engaged, both in their leisure time and at workplace. Social networking is a phenomenon that existed ever since society was formed. Human beings always sought to live in social environments. The proliferation of SNS and their pervasion in everyday life is now affecting how the youth (Gen Y) round the globe especially in India are managing their network. The willingness of users to consider SNS as a means of communication and social networking in everyday life has made it compulsory for the authorities to frame new policies. Online social networking sites like Face book, LinkedIn, Instagram, and Google Buzz are influencing the way users establish, maintain and cultivate a range of social relationships from close friendships to casual acquaintances. These social networking sites (SNS) are nothing but "websites which make it possible to form online communities and share user created content". There have been several definitions of the term "social networking" which has lead to confusion among readers. For this, SNS can be broadly defined as internet-based social spaces designed to facilitate communication, collaboration and content sharing across networks of contacts. It allows members to manage, build and represent their social networks online.

SNSs are utilized by people of all the age groups but the adolescents and youth use it to the fullest for various purposes (mainly communication). The Internet as a medium of research has changed drastically since it has become more complex, virtual, social and physical such that the youth participates and co-constructs something, rather than just simply watching on the television or personal computers. It is a complex virtual world behind that small screen on which developmental issues play out. (Deshmukh al., 2014) Online mediums also connect people in a person-to-person manner which is more direct and interpersonal. The levels of verbal and affective intimacy can be known through observing the frequency of posts on popular SNSs.(Kross et al., 2013) The popularity of social networking sites is not just based on how many people it has but on how many hours or time a user spends on it daily. Some social aspects like family pattern, age, communication among the members play a vital role in the youth's web usage. Adequate parenting plays the role of a catalyst in serving the social and emotional needs of the youth, devoid of such parenting they result in fulfilling those needs from online sites. Social media usage behaviour is developing and transforming at a rapid rate leaving its affects on the youth. (Lee, Lee, Kwon, 2011)The dark side of social Medias that its excessive usage effects all the facets of a youth's life including psychological, emotional, physical well-being and social development.

Gender differences are apparent in Social Networking Sites preferences and amount of use. The sites most popular with teenagers and young adults of both genders (as of 2014) are Facebook and Twitter, which constitute social network sites according to the three criteria articulated by Pempek (2009). They have user profiles, allow for 'friending' (or 'following' on Twitter), and contain social networks that can be navigated to encounter friends of friends. A few study 


\section{Gender Differences in Usage of Social Networking Sites and Perceived Online Social Support on Psychological Well Being of Youth}

conducted in the U.S. found that $80 \%$ of online teens use social network sites, Facebook being the most popular, with 93\% of those teens reporting its use (Mango et al., 2008) However, girls on average spend more time on social network sites and use them more actively than boys do (Burke,2010). More girls than boys use Facebook and Twitter; female users, including teens, also predominate on the online pinboard Pinterest. Conversely, more males use music-sharing sites such as last.fm, as well as Reddit, a social news website known for its sometimes misogynistic content (Kraut \& Crawford, 2012).

Gender differences are also present in the ways teens use the internet and social media, although usage patterns have shifted over time. Teenage boys in the United Kingdom reported using computers more often than girls and feeling more comfortable doing so (Liu, C.2013). However, both genders were embracing the internet as a means of communicating with their friends: Gross (2004) found that the most common activity among American middle and high school students was chatting via instant messaging. In 2007, teenage girls in the U.S. were more active bloggers than boys - perhaps the first time that females were more active participants than males in a public mode of computer-mediated communication. Boys, meanwhile, were more likely to upload online videos and use video sharing applications. Boys spend more time using computers, especially playing video games and visiting video websites such as YouTube. However, girls create and share more video and also are more likely to video chat, in keeping with their more active texting and mobile communication behaviors. Regardless of gender, most teens in the U.S. today spend part of their leisure time online visiting social media sites.

\section{Perceived Social Support:}

The information leading the subject to believe that he/she is cared for, loved, esteemed, valued and belongs to a network of communication and mutual obligation, has been believed to increase the well-being of individuals is known as "Perceived Social Support". Peers are a major source of social support for the youth today and give greater importance to earning this social support through all possible means (Barker,2010).However, the notion of perceived social support has now changed for the Gen Y due to the widespread use of SNSs (Joinson, A. Goulet, 1998). As a result, studies began to examine the relationship between SNSs usage and perceived social support among youth (Qiu and Tov, 2012).

Researchers suggest that online perceived social support has negative effects on the psychological well-being of the youth, still not many researchers have focused on the impact of perceived social support. There are positive effects of the online social support on the well-being of the youth which can be explained through "Main effects model of the social support" (Cohen and Wills, 1984). This suggests that the online social support directly affects the well-being irrespective of the amount of stress a person experiences.

\section{Well-being:}

The term well-being (WB) may be viewed as an abstract and wholly individualized concept whose meaning appears in constant flux. Well-being can be understood as "how people feel, how 


\section{Gender Differences in Usage of Social Networking Sites and Perceived Online Social Support on Psychological Well Being of Youth}

do they function, both on a personal and social level and how do they evaluate their lives as a whole". To make it simpler, how people feel is the "emotional well-being", how do they function on a social level is "social well-being". Irrespective of the psychological measure, the well-being has a strong relationship with the social support. Past studies by both Ashmore (1979) have shown association between well-being and high "relatedness" provided by social networks..A research review also concludes that in general those who have greater intimacy and higher quality relationships also have higher well-being (Berkman, 2001). The importance of social support is further emphasized when one considers the psychological costs associated with the suppression of emotions caused by limited social support. (DeNeve and Cooper,1998; King and Pennebaker,1998). Limited social support from the immediate surroundings might lead to increase in usage of SNSs in search of social support, affecting the person's well-being (Cohen, 1985).

Psychological well-being includes the ability to be autonomous, solving problems, managing emotions, experience empathy, be resilient and attentive. Psychological well-being includes six main aspects:

1) Self acceptance which refers to the positive attitude towards oneself,

2) Personal growth as the feeling of sustained development and possibilities,

3) Life purpose as to having a goal and reason for one's existence,

4) Mastery referring to a feeling of handling complex situations,

5) Positive relatedness as having positive and intimate social relationships and being interested in the well-being of others,

6) Autonomy comprising of being self-dependent and independent.

Since this classification of well-being, most of the research has being done either in on personal functioning (psychological well-being) or on global measures of life satisfaction and happiness (emotional well-being). (Naman et al., 2012)

Keeping in mind that psychological and emotional well-being are studied the most, there are certain social aspects attached to the two. For instance, studies showed that interaction with family, friends and neighbors leading to higher emotional well-being, with friends being more important than family and neighbors. Other social aspects like family pattern, marital status, trustworthiness also play an important role in determining the psychological and emotional wellbeing of people.

In this study the impact of social networking and perceived online social support on the psychological well-being among youth is measured through a survey. Here, the genders are compared and contrasted in order to obtain the difference (if at all there is any) among the impacts it has on males and females. Studies have shown that the SNS use, level of perceived social support and risk towards lower well-being vary according to the gender, but no study has 


\section{Gender Differences in Usage of Social Networking Sites and Perceived Online Social Support on Psychological Well Being of Youth}

yet examined if these concepts are related differently among males and females. Hence, this study will test if the relationship between SNS use, perceived social support and lower wellbeing differ among males and females from the age of 18 to 25 years. Research has shown that perceived social support might have a positive relationship with SNS use but negatively affects the psychological well-being. Therefore, the current study also aimed to study the relationship and mediating role of perceived social support between the SNS use and the youth's well-being.

According to social roles theory, gender differences can be understood by attending the social roles of males and females. More specifically, as a result of the social roles theory males and females learn different abilities and beliefs, depending on which they behave socially. While males are expected to be a genetic (i.e. independent, rational, competitive), women are expected to be communal (i.e. submissive, dependent and gentle). The role of the females thus favors the engagement in interpersonal activities. So, the social role theory provides a theoretical background as to why females are more attracted to social networking sites compared to males to maintain interpersonal relationships.

\section{HYPOTHESIS}

Considering the review of literature done above, the hypotheses for the present study were:

H1: Females will be more attracted to social networking sites compared to males.

H2: Females will tend to perceive more online social support compared to males.

H3: There will be a negative correlation between usage of social networking sites and psychological well-being.

H4: There will be a positive relationship between online perceived social support and psychological well-being.

\section{METHOD}

The research methodology in this study includes Participants, measurement instruments, and research procedure and data collection.

\section{Participants}

100 college students between the age of 18 to 25 years pursuing either their bachelors or masters degree constituted the sample for this research. The sample was selected through the convenient sampling method. The questionnaires were prepared using the Google form in the Google drive. The questionnaires were sent to them through E-mail and personal messages over the phone. The responses were recorded automatically in the drive. On the first day itself $70 \%$ participants responded. Responses contained 50 males and 50 females. The sample differed in age, gender, family pattern, educational qualification and name of the college/university. 


\section{Instruments}

The following instruments were used in the data collection:

1. Personal Data Sheet: This instrument (devised by the researcher) contained the demographic details like age, gender, family pattern, educational qualification and name of college/ university.

2. Social Networking Survey (Deshmukh, Tatbe ; 2014):The instrument contained 22 questions pertaining to usage hours, medium through which subjects used the sites, the social capital, number of sites used frequently.

3. Multidimensional Scale of Perceived Social Support Assessment (Zimet, Powell, Werkman \& Berkoff; 1990): This instrument had 12 items measuring the social support from online friends and family members. The target user of this instrument was the youth which is in sync with the sample of this study. The 12 items were scored on a 7 point scale. The possible range of total score is 7-84. The reliability found through Cronbach's coefficient alpha was .88 .

4. Psychological General Well-Being Index (PGWBI)( Chassany, Wu, Dupuy, 1970-71) : The 22 item PGWBI targeted peoples' self- representation of an aspect of the general well-being. It did not include an evaluation of physical health. It included six dimensions: Anxiety, Depressed mood, Positive Well-being, Self-control, General Health and Vitality. The scoring was generally on a 1-6 scale, giving the score range of 22-132. There was no reverse scoring done in this instrument.

\section{Procedure}

The entire process of data collection involved two steps as mentioned below:

1. Preparing the questionnaire: The questionnaire had to be typed in the Google form and then sent to the decided sample. All the previously mentioned instruments were used and their questions were entered.

2. Distributing the questionnaire: Once the Google form was ready, the form was e-mailed or messaged on the phone of the participants. They were asked to respond as promptly as possible. A time of one week was allotted for data collection. On the first day itself, the return rate was $70 \%$ (i.e. $70 \%$ people responded on the same day).

Later on, the responses were automatically recorded and data analysis was carried out by the researcher.

\section{Statistical Analysis}

Three data analysis methods were used in this study: descriptive statistics, correlation and one way ANOVA. The data for this research was analyzed using the Statistical Package for the Social Sciences (SPSS 16.0). 


\section{RESULTS}

For the descriptive analysis, the mean and standard deviation of all the variables, including psychological well-being, usage hours, and perceived social support was computed. This was done in order to arrange the data in a more interpretable manner. .

Table 1: Descriptive Analysis of Psychological well-being, Perceived Social Support and Usage hours

\begin{tabular}{|c|c|c|c|}
\hline \multicolumn{4}{|l|}{ Descriptive Statistics } \\
\hline Variables & $\mathrm{N}$ & Mean & Std. Deviation \\
\hline Psychological well-being(PWB) & 100 & 77.590 & 5.7719 \\
\hline Perceived Social Support (PSS) & 100 & 67.220 & 31.3590 \\
\hline Usage hours & 100 & 68.150 & 10.8845 \\
\hline
\end{tabular}

The maximum standard deviation among the three variables is of Perceived Social Support (PSS) i.e. 31.3590 which shows that the scores of PSS are the most scattered among the participants followed by usage hours (10.8845) and the least scattered scores are of PWB Usage hours (5.7719), showing that they are least deviated from the mean among all the variables.

\section{Pearson Product-Moment Correlation:}

Table 2: Correlation values of Psychological well-being and Perceived Social Support.

\begin{tabular}{|l|l|l|l|}
\hline \multicolumn{2}{|l|}{ Correlations } \\
\hline \multirow{4}{*}{ PWB } & Pearson Correlation & PWB & PSS \\
\cline { 2 - 4 } & Sig. (2-tailed) & 1 & -- \\
\hline \multirow{4}{*}{ PSS } & $\mathrm{N}$ & & \\
\cline { 2 - 4 } & Pearson Correlation & 100 & \\
\cline { 2 - 4 } & Sig. (2-tailed) & $.230^{*}$ & 1 \\
\cline { 2 - 4 } & $\mathrm{N}$ & .019 & 100 \\
\hline \multirow{2}{*}{ *. Correlation is significant at the 0.05 level (2-tailed). } & 100 \\
\hline
\end{tabular}

Pearson Product moment correlation was computed to examine the correlation between the independent and dependent variables. The values are depicted in the table above. According to the results of correlation analysis, the correlation between psychological well-being (PWB) and perceived social support (PSS) was 0.230 which is significant at 0.05 level. $[r=.230, N=100, p$ $=.019]$. It shows that with the increase in PSS, the PWB also increases and vice versa. The results show a significant positive correlation between the two variables. 


\section{Gender and Perceived Social Support (PSS):}

Table 3: The one way ANOVA table depicting the relationship between gender and perceived social support (PSS).

\begin{tabular}{|l|l|l|l|l|l|}
\hline PSS \\
\hline & Sum of Squares & df & Mean Square & F & Sig. \\
\hline Between Groups & 1466.890 & 1 & 1466.890 & 4.009 & .003 \\
\hline Within Groups & 10261.860 & 98 & 104.713 & & \\
\hline Total & 11728.750 & 99 & & & \\
\hline
\end{tabular}

a. Predictor: Gender

b. Dependent Variable : PSS

One way analysis of variance was carried out to find the significance of difference between gender groups and Perceived Social support on study variables. The results of ANOVA for gender groups are presented in Table (1). The results revealed significant differences between males and females on Perceived Social support $(\mathrm{F}=4.009, \mathrm{p}<.05)$.

\section{Gender and Usage hours:}

Table 4: The one way ANOVA table depicting the relationship between gender and Usage hours.

\begin{tabular}{|l|l|l|l|l|l|}
\hline & Sum of Squares & df & Mean Square & F & Sig. \\
\hline Between Groups & 1.960 & 1 & 1.960 & 5.418 & .038 \\
\hline Within Groups & 43.480 & 98 & .444 & & \\
\hline Total & 45.440 & 99 & & & \\
\hline
\end{tabular}

\section{a. Dependent Variable : Usage Hours \\ b. Factor: Gender}

One way analysis of variance was carried out to find the significance of difference between gender groups and Usage hours on study variables. The results of ANOVA for gender groups are presented in Table (2). The results revealed significant differences between males and females on Usage hours $(\mathrm{F}=5.418, \mathrm{p}<.05)$.

\section{DISCUSSION}

The research aims at studying the effects of usage of social networking sites and perceived online social support on the psychological well-being among the adolescents. The social networking has been a very important part in the students' life. In India, not many studies have been done on this topic. The researcher here has tried to relate online perceived social support 


\section{Gender Differences in Usage of Social Networking Sites and Perceived Online Social Support on Psychological Well Being of Youth}

and psychological well-being as the adolescents' psychological well-being has become a matter of concern in the last few decades. The students include participants from 18 to 25 years. The variables like usage of social networking sites and online perceived social support were compared among males and females. A positive correlation between the perceived online social support and psychological well-being was found using Pearson's Product-Moment Correlation. One-way ANOVA was also computed to find out differences among males and females in the study. Other variables like family pattern and usage hours of the SNS were shown in the form of graphs and pie charts.

Online Social support has been one of the important functions of Social Networking (Gottlieb, 2000). Studies by Kraut et. al. showed that there was a relationship between perceived social support and psychological well-being. The online perceived social support was found to affect the psychological well-being of the subjects. "The Internet Paradox Revisited" reaffirmed the relationship between SNS usage and social support further affecting the psychological wellbeing. Similarly, social theory reviewed in the literature lead the researcher to find gender differences among each of the variable.

\section{H 1: Females will be more attracted to social networking sites compared to males.}

Early research shows that "computer culture" was difficult for the women to cope up with but gradually they came at par with the males. Many researchers like Valenzuela, Tufekci (2008, 2009) found considerable differences between the usage patterns and purposes of the usage of SNS among males and females. Females used it to maintain existing relationships while males used the SNS for games, developing new contacts. Females are more prone to SNS as compared to males and spend more hours daily on the SNS. In the present study, One-way ANOVA was applied to find if the usage hours varied among males and females. The obtained value of $\mathrm{F}$ (statistic) was significant with a $\mathrm{p}<0.05$.(Table: 7 ). Theoretically, the social role theory might also be the reason for females to be attracted to social media. This theory suggests that due to different social roles, they also learn different skills and beliefs which in turn form their social behavior. The female gender is expected to be more communal while the male gender is more a genetic and independent. Hence, the results support the first hypotheses that females are more attracted to social networking sites compared to males.

\section{H 2: Females will tend to perceive more online social support as compared to males.}

Both the types of online social support (i.e. perceived and received) were studied in many early researches. As college students move away from homes, they heavily rely on internet for social support. Studies have shown that girls specially perceive and receive more social support as they utilize more social networking sites and are found to be more extroverted compared to males (Frison \& Eggermont ; 2015). In the present study also the results have reaffirmed the conclusions of the previous researches but here only "perceived online social support" is taken into account. The one-way ANOVA was applied to check the effect of gender on perceived 


\section{Gender Differences in Usage of Social Networking Sites and Perceived Online Social Support on Psychological Well Being of Youth}

online social support. The obtained value of $\mathrm{F}$ (statistic) is 14.009 which is higher than the critical value of $\mathrm{F}$ at the $(1,98)$ degree of freedom. The value of $\mathrm{F}$ obtained is significant at 0.01 level $(p=0.003)$. (Table: 5). The probable reason for this is that males and females have different self-representation concerns -females are more concerned about the image they represent and how they are perceived by others while males are less or not bothered about it in face to face and online conversations both. The results here support the hypotheses and we accept the hypotheses.

\section{H 3: There will be a negative correlation between social networking sites and psychological well-being.}

Earlier research found a positive relation between usage of SNS and psychological well-being. According to the main effects model the usage hours of SNS did affect the psychological wellbeing. The theory states that the integration into the social network might have positive effects on the psychological well-being. Contrary to these findings, study by Moody (2001) showed that greater usage of social networking sites lead to a decline in the psychological well-being. Further, the results indicated that uncontrolled use of the SNS would lead to depression, loneliness etc. A study in 2010 showed that hyper-texting and hyper-networking led to the subjects indulging in alcoholism, smoking and finally declining the psychological well-being among college going students (Consumer Reports, 2010). In the present study, the results show a negative correlation between usage of social networking sites and psychological well-being. The value of $\mathrm{F}$ was statistically significant at the level of $0.05(\mathrm{p}=0.041)$ (Table: 8).The youth today cannot strike a balance between the surrounding real environment and the virtual online world. Due to this reason, they are continuously trying to take out time for both and end up with a decline in the psychological well-being. Their academics is also affected due to this poor management and excessive usage of SNS leads to depression, stress etc. Thus with the increase in the usage hours, the psychological well-being will keep on decreasing. Hence, the hypotheses was statistically proven and accepted by the researcher.

\section{H 4: There will be a positive relationship between online perceived social support and psychological well-being.}

The review of literature states that online social support perceived and received from the online contacts increase life satisfaction and psychological well-being, buffering the negative effects of stress (Cauce et. al.,1994 ; Meeus, 1994; Robinson, 1995). Shaw and Grant (2000) also found that depression and loneliness decreased when people indulged in social networking activities due to the online social support. SNS affects social support and psychological well-being way faster than it affects the self-esteem of the participants. In the present study, only the correlation between the two variables was found. The Pearson's correlation between perceived social support and psychological well-being was .230 which was found to be significant at the level of 0.05. ( $\mathrm{p}=0.019, \mathrm{~N}=100)$ (Table: 4) Theoretically, "rich gets richer" hypotheses is apt among today's youth. It says that people who are already getting social support will get more and more 


\section{Gender Differences in Usage of Social Networking Sites and Perceived Online Social Support on Psychological Well Being of Youth}

social support while those who are discouraged as they have no social support would have a declined rate of psychological well-being leading to depression. Since the results show positive correlation, the psychological well-being will increase with every increase in the online perceived social support and vice versa.

\section{LIMITATIONS}

In the present study, only the usage hours of SNS were taken into consideration but the researcher could have studied more variables pertaining to the social networking sites like purposes of usage, most popular websites, and social capital. The sample size could have been more in order to get more in order to get more reliable results but due to time constraint it was not done in this study. The interaction effect between online usage hours and perceived social support on psychological well-being was not seen in this study. Questionnaires used could have been complimented with a few interviews having open ended questions and analysis of conversations in order to get a deeper insight in the matter.

\section{IMPLICATIONS FOR FURTHER RESEARCH AND STUDY}

The current study looked at the effect of social networking sites and online perceived social support on the psychological well-being of youth. Here, certain important relationships were established one of the most important one is "the negative relationship between usage hours of SNS and psychological well-being" - it acts as an alarming sign for the adolescents and the future generations too. More studies and researches need to be done in this area especially in the Indian context with a view to increase awareness among the population. As mentioned, in the literature review that the self-esteem, online social support is predictors of psychological wellbeing of an individual so more studies need to be made in that context. The contribution of current research is that the researcher has found that perceived social support at many times plays the role of mediating the relationship between usage hours of SNS and the psychological well-being and gender differences play a role in the relationship between these variables.

\section{CONCLUSION}

The study showed a positive relationship between online perceived social support and psychological well-being while a negative correlation was observed between the usage hours and psychological well-being of the participants. Also significant gender differences were obtained in certain variables like daily usage and online perceived social support. Thus, a limited usage of the SNS would be beneficial to the adolescents.

\section{REFERENCES}

Allen, J.P., Evans, M.A., Hare, A.L., \& Mikami, A.Y. (2010). Adolescent Peer Relationships and Behavior Problems Predict Young Adults' Communication on Social Networking Websites. Developmental Psychology, 46(1), 46-56. doi: 10.1037/a0017420 


\section{Gender Differences in Usage of Social Networking Sites and Perceived Online Social Support on Psychological Well Being of Youth}

Ashmore,R.D.,(1979).Sex Stereotypes and Implicit Personality Theory: Toward a Cognitive Social Psychological Conceptualization. Sex Roles, 5, 219-248.

Baker, R. K., \& White, K. M. (2010). In their own words: Why teenagers don't use social networking sites. Cyberpsychology, Behavior and Social Networking, 14(6), 395-398.

Berkman F. (2001). Social Ties and Mental Health. Journal of Urban Health : Bulletein of the New York Academy of Medicine, 78, 458-467.

Boyd, D.M., \& Ellison, N.B. (2007). Social network sites: Definition, history and scholarship. Journal of Computer-Mediated Communication, 13(1), 210-230.

Burke M. , Marlow C. , Lento T. (2010). Social Network Activity and Social Well-Being. CHI'10.

Cohen, S., \& Ashby Wills, T. (1985). Stress, social support and the buffering hypothesis. Psychological Bulletin, 92(2), 310-357.

DeNeve, K. M., \& Cooper, H. (1998). The happy personality: A meta-analysis of 137 personality traits and subjective well-being. Psychological Bulletin, 124, 197-229.

Deshmukh P. , Deshmukh S. , Tathe C. (2014). An Impact Of Social Networking Sites On Youngsters. Journel Impact Factor, 5(12), 24-35.

Developmental Psychology. 25,633-649.

Gross, E.F., 2004. Adolescent Internet use: What we expect, what teens report. Applied

Joinson, A. (1998). Causes and implication of disinhibited behavior on the Intrnet. Psychology and the Internet: intrapersonal, interpersonal, and transpersonal implications, 43-60.

Kraut, R., Kiesler, S., Boneva, B., Cummings, J., Helgeson, V., \& Crawford, A. (2002). Internet paradox revisited. Journal of Social Issues, 58, 49-74.

Kross E, Verduyn P, Demiralp E, Park J, Lee DS, et al. (2013). Facebook Use Predicts Declines in Subjective Well-Being in Young Adults. PLoS ONE, 8(8).

Lee, G. L. (2011). Use of social-networking sites and subjective well-being: A study in South Korea. Cyberpsychology, Behavior and Social Networking, 14, 151-155.

Liu, C. \&. (2013). Can Facebook use induce well-being? Cyberpsychology, Behavior and Social Networking, 16, 674-678.

Manago, A. M., Taylor, T., \& Greenfield, P. M. (2012). .Me and My 400 Friends:The Anatomy of College Students' Facebook Networks, Their Communication Patterns, and Well-Being. Developmental Psychology. Advance online publication.

Naaman M. , Brody S. , Swaine F. (2012). Of Joy and Gender : Emotional Expression in Online Social Networks. CSCW'12, 1-4.

Pempek, T. A., Yermolayeva, Y. A., \& Calvert, S. L. (2009). College students' social networking experiences on Facebook. Journal of Applied Developmental Psychology, 30, $227-238$.

Qiu, L., Lin, H., Leung, A. K., \& Tov, W. (2012). Putting their best foot forward: Emotional disclosure on Facebook. Cyberpsychology, Behavior and Social Networking, 15, 569572. 